\title{
Impairment of self-monitoring:
}

\section{part of the endophenotypic risk for psychosis}

\author{
DAGMAR VERSMISSEN, INEZ MYIN-GERMEYS, ILSE JANSSEN, \\ NICOLAS FR ANCK, NICOLAS GEORGIEFF, JOOST a CAMPO, \\ RON MENGELERS, JIM VAN OS and LYDIA KRABBENDAM
}

\begin{abstract}
Background A disorder of selfmonitoring may underlie the positive symptoms of psychosis. The cognitive mechanisms associated with these symptoms may also be detectable in individuals at risk of psychosis.
\end{abstract}

\begin{abstract}
Aims To investigate (a) whether patients with psychosis show impaired selfmonitoring, (b) to what degree this is associated with positive symptoms, and (c) whether this is associated with liability to psychotic symptoms.
\end{abstract}

\begin{abstract}
Method The sample included: individuals with a lifetime history of non-affective psychosis ( $n=37)$, a genetically defined risk group $(n=4 \mathrm{I})$, a psychometrically defined risk group ( $n=40)$, and control group $(n=49)$. All participants carried out an actionrecognition task.
\end{abstract}

\section{Results Number of action- recognition errors was associated with psychosis risk (OR linear trend over 3 levels: I.12,95\% CII.04-1.20) and differential error rate was associated with the degree of delusional ideation in a dose-response fashion (OR linear trend over 3 levels: I.13,95\% Cl I.00-1.26).}

\section{Conclusions Alterations in self-} monitoring are associated with psychosis with evidence of specificity for delusional ideation. In the risk state, this is expressed more as failure to recognise self-generated actions, whereas in illness failure to recognise alien sources come to the fore.

Declaration of interest None.
Source-monitoring refers to different processes involved in ascribing memories, knowledge and beliefs to a certain source of information (Johnson et al, 1993; Henquet et al, 2005). Self-monitoring is a specific type of source-monitoring, namely the capacity to distinguish the consequences of self-generated items from those that are externally generated. The hypothesis that alterations in self-monitoring underlie the positive symptoms of psychosis has been put forward by different authors (Bentall, 1990; Johns et al, 2001). For example, the phenomenon that people attribute selfgenerated actions to an external cause, one of the first-rank symptoms of psychosis according to Schneider (1959), is possibly due to impaired self-monitoring. Defective monitoring may be the outcome of a bottom-up deficit, specifically, a lack of awareness of the predicted consequences of self-generated actions (Frith et al, 2000), in combination with a top-down externalising bias, which makes someone prone to ascribing erroneously actions to an external agent (Allen et al, 2004).

The current study aimed to examine potential deficits in the bottom-up process. When monitoring a self-generated action, the individual has to match the consequences of the executed movement to the internal representation. In order to test the hypothesis that this process is flawed in psychosis, Franck and colleagues developed a procedure allowing the experimenter to distort performed actions with respect to the participant's own movement (Franck et al, 2001). It was shown that patients with psychosis had difficulties recognising their own actions compared with the control group (Franck et al, 2001).

An essential issue is whether the psychological mechanisms of psychosis can also be shown to operate in individuals at risk but without current clinical need (Bentall, 1990; Janssen et al, 2003). Firstdegree relatives of individuals with psychosis (higher than average genetic risk) and individuals with subclinical psychotic experiences in the general population (higher than average psychometric risk) are examples of such risk groups (Claridge, 1994). Evidence for a deficit in self-monitoring in individuals at risk would imply that such a deficit is associated not only with the expression of the phenotype, but also with transmission of risk.

The current study, therefore, included four groups with different levels of vulnerability to psychosis: (a) patients with a lifetime history of non-affective psychosis, (b) a genetic risk group of non-psychotic first-degree relatives of individuals with a lifetime history of non-affective psychosis, (c) a psychometric risk group of healthy subjects from the general population with a higher than average level of positive psychotic experiences and (d) well controls from the general population, with the aim of extending the findings by Franck and colleagues (2001). The hypothesis was tested that (a) patients with non-affective psychosis show impaired monitoring of their actions, (b) this alteration is also present in individuals at risk, albeit to a lesser degree, and (c) this alteration is conditional on the presence of positive psychotic symptoms or experiences.

\section{METHOD}

\section{Participants}

Four groups differing in the degree of vulnerability to psychosis were sampled in the Cognitive functioning in Psychosis $(\mathrm{CoP})$ study: (a) individuals with lifetime history of non-affective psychosis, (b) first-degree relatives of individuals with non-affective psychosis, (c) healthy subjects scoring high ( $>75$ th percentile) on the positive dimension of psychosis-proneness measured by the Community Assessment of Psychic Experiences (CAPE; Hanssen et al, 2003) and (d) healthy controls, i.e. participants scoring in the average range (40-60th percentile) on the CAPE. All participants were between the ages of 18 and 55 years, sufficiently fluent in Dutch and without a history of central neurological disorders. Written informed consent in accordance with the local ethical committee guidelines was obtained from all participants.

Participants were recruited from the catchment area community mental health centre (source population of 350000 ) and the catchment area psychiatric hospital. Initial inclusion criteria for participants 
with psychosis were lifetime prevalence of a period of psychosis in clear consciousness, according to the Research Diagnosis Criteria. Relatives were sampled through participants with psychosis or through associations for relatives of individuals with psychotic symptoms. Participants with average and high levels of psychotic experiences were recruited from a general population sampling frame in the city of Sittard (Hanssen et al, 2003). Participants, 2287 females and 2302 males, had been randomly selected and sent a letter in which they were asked to participate. In addition to the participants themselves, family members were also invited to participate using a snowball-sampling procedure. A total of 765 subjects aged 17-77 years, pertaining to 374 families filled in the CAPE. The subjects with a mean (i.e. between 40th and 60th percentile) and a high (i.e. above 75th percentile) score on the CAPE positive psychosis dimension were invited to participate in the CoP study. The CAPE (http://www.cape42.homestead.com) is a self-report questionnaire designed to assess dimensions of the subclinical psychosis phenotype. It includes dimensions of positive $(n=20)$ and negative $(n=14)$ psychotic experiences as well as depressive experiences $(n=8)$.

The study sample included 45 individuals with psychosis (44\% in-patients), 47 first-degree relatives with no psychosis, 41 healthy participants with a high level of psychotic experiences and 54 healthy controls with an average level of psychotic experiences. Of the 47 healthy relatives there were 13 mothers, 8 fathers, 15 sisters, 8 brothers, 2 daughters and 1 son. Twentyseven families contributed at least 1 patient and 1 relative. Four relatives participated without their family member with psychosis.

For all participating patients, the Operational Criteria Checklist for Psychotic Disorder (OCCPI) was completed based on case note material and Positive and Negative Syndrome Scale (PANSS) interview. Where necessary, additional information was derived from ward staff or case-managers. Using the information in the OCCPI, the computerised program OPCRIT yielded Research Diagnosis Criteria diagnoses.

\section{Instruments}

Action-Recognition Task

An action-recognition task designed by Franck et al (2001) was carried out by all subjects. A stillage held a computer screen that was placed face down. Images on this computer screen were reflected in a horizontal mirror, placed $18 \mathrm{~cm}$ below the screen and $31 \mathrm{~cm}$ above a table at which the participant was seated. A joystick was placed on the table. The image of a virtual hand was presented in the mirror, superimposed on the participant's own hand. This setting enables participants to actually move the joystick, while being exclusively exposed to the image of the virtual hand, moving analogously to their own. Participants sat on a chair in front of the setting, their forehead resting on a foam cushion which enveloped the metallic stillage. The joystick had to be held with the hand of preference, which was in all cases the right hand, while the elbow rested on the table. Participants executed a series of discrete movements with the joystick. A green spot was displayed for a brief moment (1s) on the left, the right or the top of the screen. In the following $2 \mathrm{~s}$ the virtual hand was brought into vision. During these $2 \mathrm{~s}$, participants had to move the joystick in the direction indicated before by the green spot. Simultaneously, a movement of the virtual hand was shown. Participants had to decide whether the movement they saw on the screen was an exact copy of the movement they had made. This decision had to be made immediately after each trial and could be revealed verbally by a simple 'yes' or 'no'.

The task consisted of 120 trials that were divided into three categories: (a) 24 neutral trials in which the virtual hand made an exact copy of the actual movement made by the participant, (b) 48 trials with temporal biases, and (c) 48 trials with angular biases. The temporal biases, in which the movement of the virtual hand was delayed by a fixed time $(100,200$, 300 or $400 \mathrm{~ms}$ ) compared to the actual movement, and angular biases, in which the movements of the virtual hand deviated by a specific angular value $\left(10^{\circ}, 20^{\circ}, 30^{\circ}\right.$, $40^{\circ}$ ) with regard to the point of comparison, were randomly introduced.

Before the actual task started, participants had the opportunity to familiarise themselves with the techniques to handle a joystick. During this training session an angular bias, temporal bias and neutral trial were presented.

\section{Present State Examination}

The purpose of the Present State Examination (PSE; Wing et al, 1974) is to assess the presence and severity of symptoms associated with a broad range of major psychiatric disorders over a designated period, i.e. the past week, by means of a structured clinical cross-examination of the individual. In this study, only the sections that cover signs and symptoms of psychotic disorders were used (43 items: PSE 55-92, plus their sub-scale scores).

\section{General intelligence}

General intelligence was measured by a combined score on one performance subtest and one verbal sub-test from the Groninger Intelligence Test (GIT; Luteijn \& van der Ploeg), a widely used Dutch intelligence test. This test yields results that are comparable to those of the Wechsler Adult Intelligence Scale - Revised.

\section{Analyses}

Statistical analyses were carried out using STATA version 9.1. A four-level group variable was constructed reflecting the risk for psychosis in order of a priori hypothesised strength with value 3 for participants with psychosis, 2 for genetic risk, 1 for psychometric risk and 0 for controls. Two types of errors could potentially be made (a) participants misidentifying a neutral trial as different from their own action and (b) participants misidentifying trials with temporal or angular biases as similar to their own movement. Errors of this latter type could be subdivided into angular biases and temporal biases. Results of subjects who gave the same answer in $90 \%$ or more of the total number of trials were excluded from the analyses.

Utilizing the XTGEE module in STATA, a multilevel approach to logistic regression was used to assess the association between making an error on the action-monitoring task and the four-level group variable for psychosis risk. The association between committing an error on the action-monitoring task and symptomatology was similarly established with additional adjustment for group. Multilevel random effects modelling techniques are a variant of the more often used unilevel regression analyses and are ideally suited for the analysis of data in which repeated observations (120 action-monitoring trials) are nested within participants. All analyses were $a$ priori adjusted for age and gender. In order to examine dose-response relationships between symptomatology and errors on the action-monitoring task, the group 
was divided into three groups according to their tertile level of symptom score. Odds ratios (OR) with their $95 \%$ confidence intervals $(95 \% \mathrm{CI})$ were used to express effect sizes, with the control group as the reference category.

\section{RESULTS}

\section{Sample characteristics}

The action-recognition task was carried out by 36 participants with non-affective psychosis, 40 non-psychotic first-degree relatives of participants with non-affective psychosis, 39 healthy participants from the general population scoring high ( $>75$ th percentile) on the positive dimension of psychosis-proneness measured by the CAPE and 49 healthy control participants with an average score on the positive dimension of the CAPE. Missing data were due to technical problems $(n=23)$. Two participants, 1 participant with psychosis and 1 participant with subclinical psychotic experiences, were excluded from the analyses since they gave directionally persistently similar answers in at least $90 \%$ of all trials. The participants with psychosis group, in which men were overrepresented $(78 \%)$, was younger than the other groups (Table 1). According to the OPCRIT, there were 27 participants with psychosis $(75 \%)$ with a Research Diagnostic Criteria diagnosis of schizophrenia, 3 patients $(8 \%)$ with a diagnosis of schizoaffective disorder, 5 patients $(14 \%)$ with a diagnosis of unspecified functional psychosis and $1(3 \%)$ for whom OPCRIT data were missing but who had a clinical diagnosis of non-affective psychotic disorder.

\section{Action-monitoring and psychosis risk}

The number of errors in all trials combined increased progressively with risk group, although differences between the risk groups and controls were small (controls: 29.7\%; psychometric-risk group: $31.1 \%$; genetic-risk group: $31.8 \%$; participants with psychosis: $37.4 \%$; OR linear tren$\mathrm{d}=1.12$, 95\% CI 1.04-1.20). The separate ORs relative to the controls were: 1.06 (95\% CI $0.85-1.32$ ) for the psychometricrisk group, 1.17 (95\% CI 0.94-1.45) for the genetic-risk group and $1.41(95 \% \mathrm{CI}$ 1.14-1.76) for the patient group.

The proportion of errors made, for each group, in the different bias-conditions are shown in Fig. 1. In trials with a temporal bias, the error rate of the participants with psychosis group was on average $15.4 \%$ higher than the error rate of the control group.

The proportion of errors made for each of the four groups in the neutral condition is shown in Fig. 2.

After subdividing errors in trials with temporal delays, trials with an angular bias and neutral trials respectively, a significant difference between the participants with psychosis and the control group was seen in the trials with temporal delays $(\mathrm{OR}=2.40,95 \%$ CI $1.44-3.97)$, but not in neutral trials, nor in trials with angular biases (see Table 2). Differences between the genetic-risk group and controls were

Table I Sample characteristics and Present State Examination Scores for all groups

\begin{tabular}{|c|c|c|c|c|c|c|c|c|}
\hline & \multicolumn{2}{|c|}{$\begin{array}{l}\text { Controls } \\
(n=49)\end{array}$} & \multicolumn{2}{|c|}{$\begin{array}{l}\text { Psychometric-risk } \\
\text { group }(n=40)\end{array}$} & \multicolumn{2}{|c|}{$\begin{array}{l}\text { Genetic-risk group } \\
\qquad(n=4 I)\end{array}$} & \multicolumn{2}{|c|}{$\begin{array}{l}\text { Patients } \\
(n=37)\end{array}$} \\
\hline & Mean & s.d. & Mean & s.d. & Mean & s.d. & Mean & s.d. \\
\hline Age & 46.7 & 7.9 & 44.1 & 9.8 & 40.4 & II.I & 31.9 & 10.3 \\
\hline Education' & 5.6 & 0.8 & 4.9 & 1.4 & 5.4 & 1.2 & 4.5 & 1.2 \\
\hline Intelligence ${ }^{2}$ & 7.46 & 1.37 & 6.84 & 1.43 & 7.18 & 1.65 & 6.14 & 1.82 \\
\hline PSE delusions ${ }^{3}$ & 0.00 & 0.00 & 0.60 & 1.85 & 0.36 & 1.67 & 11.90 & 10.58 \\
\hline $\begin{array}{l}\text { PSE hallucina- } \\
\text { tions }^{4}\end{array}$ & 0.02 & 0.14 & 1.01 & 3.03 & 0.36 & 1.18 & 5.48 & 6.79 \\
\hline PSE $^{5}$ & 0.02 & 0.14 & 1.61 & 4.26 & 0.72 & 2.76 & 17.37 & 15.44 \\
\hline
\end{tabular}

PSE, Present State Examination.

I. Education was measured on an eight-point scale from primary school to university degree.

2. Intelligence was measured on a 10 -point scale, derived from 2 sub-tests from the Groninger Intelligence Test.

3. PSE delusions summary score (range $0-8 \mathrm{I}$ ).

4. PSE hallucinations summary score (range $0-33$ )

5. PSE psychosis summary score (range 0-II4).

highest for errors in the neutral condition $(\mathrm{OR}=2.09$, 95\% CI 1.02-4.27). In the participants with psychosis group, the number of errors in the neutral condition was also higher than in controls, but this difference failed to reach statistical significance (Table 2).

\section{Action-monitoring and psychotic symptoms}

In all trials combined, there was an association between errors and delusional ideation measured by the PSE $(\mathrm{OR}=1.13,95 \% \mathrm{CI}$ 1.00-1.26). The strength of this association between errors and delusional ideation, adjusted for group, was highest for the highest level of delusional ideation (moderate level: $\mathrm{OR}=0.93,95 \% \mathrm{CI}=0.72-1.20$ and severe level: $\mathrm{OR}=1.36,95 \% \mathrm{CI}=1.06-1.73$ ) compared with the level of no delusional ideation. No associations were found between errors and the continuous hallucinationsub-scale on the PSE $(\mathrm{OR}=1.00,95 \% \mathrm{CI}$ 0.97-1.02), nor with the continuous total PSE psychosis score $(\mathrm{OR}=1.00,95 \% \mathrm{CI}$ 0.99-1.01) overall groups.
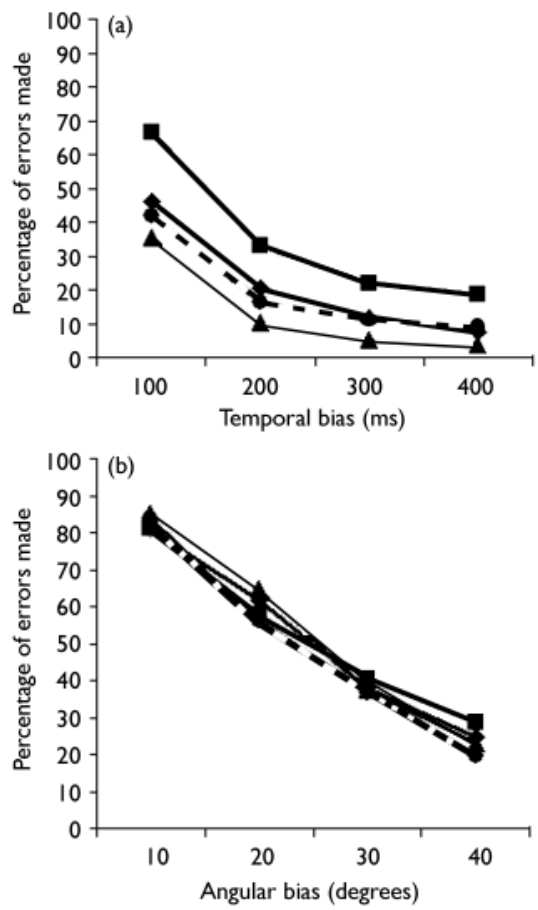

Fig. I Proportion of errors in the actionmonitoring task in matching the movement on the computer screen to own movement with conditions of (a) temporal and (b) angular bias. - - - -, controls; $\longrightarrow$, psychometric-risk group; $-\boldsymbol{\Delta}-$, geneticrisk group; - - , patients. 


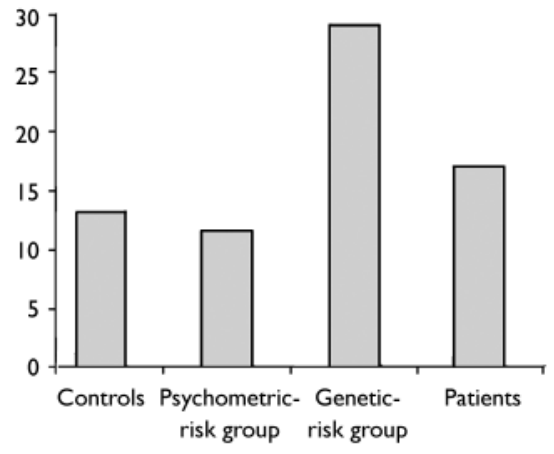

Fig. 2 Proportion of errors in action-monitoring task with neutral conditions for all groups.

\section{DISCUSSION}

The results of our study are in agreement with the findings of Franck and colleagues (2001) and suggest that alterations in selfmonitoring may be part of the endophenotypic risk to psychosis. Furthermore, the findings support the hypothesis that a selfmonitoring deficit is associated with a phenotype characterised by delusional ideation, which is in accordance with conclusions from previous studies that all found an association between alterations in self-monitoring and positive psychotic symptomatology (Bentall, 1990; Johns et al, 2001).

\section{Temporal versus angular bias}

As shown in Fig. 1 there are few or no differences between the participant groups in trials with an angular bias. These findings are partially in agreement with the conclusions of Franck et al (2001) who similarly found no significant differences between the general participants with schizophrenia and the control group. Only dissimilarities between the participants with psychosis group with delusions of influence and the control group were observed. However, differences between the groups were more apparent in the temporal trials. This difference may point to the underlying mechanism, given the fact that cerebral processing of temporal and geometric self-monitoring information is likely to involve, at least in part, different areas. Previous works have demonstrated general alterations in temporal orientation (Crow \& Stevens, 1978; Franck et al, 2005) in individuals with schizophrenia. These disturbances could underlie the high number of errors by participants with psychosis in trials with temporal bias.

\section{At-risk groups}

The use of an ordinal risk group variable is common (Miller et al, 2001; Janssen et al, 2006), and has face validity, given the fact that both genetic and psychometric at-risk groups have been shown to share phenotypic and endophenotypic characteristics as well as common risk factors with the clinical phenotype (Claridge, 1994; Faraone et al, 2000; Spauwen et al, 2004) and that over time transitions from psychometric risk state to clinical phenotype are frequent (Chapman et al, 1994; Poulton

Table 2 Results for each action-monitoring condition in three groups with different risk for psychosis (relative to healthy control group)

\begin{tabular}{|c|c|c|c|c|c|c|}
\hline \multirow[b]{2}{*}{$\begin{array}{l}\text { Action-monitoring } \\
\text { condition }\end{array}$} & \multicolumn{2}{|c|}{$\begin{array}{l}\text { Psychometric-risk group } \\
\qquad(n=40)\end{array}$} & \multicolumn{2}{|c|}{$\begin{array}{l}\text { Genetic-risk group } \\
\qquad(n=4 I)\end{array}$} & \multicolumn{2}{|c|}{$\begin{array}{l}\text { Patients } \\
(n=37)\end{array}$} \\
\hline & OR' & $95 \% \mathrm{Cl}$ & OR & $95 \% \mathrm{Cl}$ & OR & $95 \% \mathrm{Cl}$ \\
\hline \multicolumn{7}{|l|}{ Neutral } \\
\hline adjusted $\mathrm{I}^{2}$ & 0.89 & $0.38-2.06$ & 2.09 & $1.02-4.27$ & 1.26 & $0.57-2.79$ \\
\hline adjusted $2^{3}$ & 0.85 & $0.36-2.03$ & 2.06 & $1.00-4.27$ & 1.19 & $0.98-1.01$ \\
\hline \multicolumn{7}{|l|}{ Angular } \\
\hline adjusted $\mathrm{I}^{2}$ & $\mathrm{I} .14$ & $0.80-1.63$ & 1.22 & $0.84-1.78$ & 1.19 & $0.82-1.72$ \\
\hline adjusted $2^{3}$ & 1.12 & $0.77-1.61$ & 1.21 & $0.83-1.77$ & 1.13 & $0.76-1.69$ \\
\hline \multicolumn{7}{|l|}{ Temporal } \\
\hline adjusted $\mathrm{I}^{2}$ & 1.25 & $0.74-2.11$ & 0.70 & $0.38-\mid .31$ & 2.40 & $1.44-3.97$ \\
\hline adjusted $2^{3}$ & 1.10 & $0.65-1.88$ & 0.68 & $0.37-1.26$ & 1.76 & $1.02-3.04$ \\
\hline
\end{tabular}

I. OR relative to healthy control group.

2. Adjusted I, adjusted for age and sex.

3. Adjusted 2 , additionally adjusted for general intelligence.

et al, 2000). There was evidence for an overall action-recognition bias in the analyses using the ordinal risk variable, and analyses with the risk groups separately indicated intermediate positions for the latter, suggesting the presence of dose-response inherent to the ordinal risk hypothesis.

Between the genetic-risk group on the one hand and both the psychometric-risk group and the participants with psychosis group on the other, a dissimilarity with regard to errors made in different conditions could be observed. There is no direct explanation for the significant effect size of misidentifying one's own actions in the genetic-risk group, compared to the bias in recognising movements dissimilar to their own in the participants with psychosis group. As the difference between the genetic-risk group on the one hand and the participants with psychosis group on the other is the expression of psychosis, one hypothesis is that misidentifying one's own actions, similar movements, reflects a vulnerability effect, whereas bias in recognising movements dissimilar to their own is more reflective of an illness effect. Comparable observations concerning dissimilarities between vulnerability and illness effects were described in previous work on neuropsychological alterations. Verbal memory, abstraction, language functions and attention were more affected in people with the psychosis phenotype. Spatial memory, spatial abilities and sensory-motor functions, on the other hand, were more strongly affected in the genetic-risk group (Cannon et al, 1994).

Conceivably, the bottom-up process that was investigated can be controlled and dominated by a top-down mechanism, such as attribution style. It has been shown that individuals in the psychosis spectrum use an external attribution style for negative events (Fear et al, 1996; Bentall et al, 2001). Even though an externalising bias could not be observed in first-degree relatives using the Internal, Personal and Situational Attribution Questionnaire (Janssen et al, 2006), the possibility remains that deviant attribution styles are only observable in at-risk groups when measured in a less explicit way. In all likelihood, first-degree relatives, being aware of the concept of vulnerability and heightened risk for which they are examined, experience participating in a study about psychotic illness as an unpleasant, negative event and therefore develop the tendency to use external attributions resulting in a higher 
observed number of misidentifications of their own actions.

\section{Conclusions}

Alterations in self-monitoring are associated with psychosis with evidence of specificity for delusional ideation. In the risk state, this is expressed more as failure to recognise self-generated actions, whereas in the illness state failure to recognise alien sources comes to the fore. To the extent that certain delusions are the result of a failure in the identification of the source of an action in combination with an externalising attribution bias, they may be amenable to change by psychological therapies, such as cognitive-behavioral therapy (CBT). The effectiveness of CBT with respect to the treatment of delusions has been proved in previous research (Gould et al, 2001).

\section{REFERENCES}

Allen, P. P., Johns, L. C., Fu, C. H., et al (2004)

Misattribution of external speech in patients with hallucinations and delusions. Schizophrenia Research, 69, 277-287.

Bentall, R. P. (1990) The illusion of reality: a review and integration of psychological research on hallucinations. Psychological Bulletin, 107, 82-95.

Bentall, R. P., Corcoran, R., Howard, R., et al (200I) Persecutory delusions: a review and theoretical integration. Clinical Psychological Review, 2I, I143-1192.

Cannon, T. D., Zorrilla, L. E., Shtasel, D., et al (1994) Neuropsychological functioning in siblings discordant for schizophrenia and healthy volunteers. Archives of General Psychiatry, 5I, 65I-66I.

Chapman, L. J., Chapman, J. P., Kwapil, T. R., et al (1994) Putatively psychosis-prone subjects 10 years later. Journal of Abnormal Psychology, 103, 17I-183.

Claridge, G. (1994) Single indicator of risk for schizophrenia: probable fact or likely myth? Schizophrenia Bulletin, 20, 15I-168.

Crow, T. J. \& Stevens, M. (1978) Age disorientation in chronic schizophrenia: the nature of the cognitive deficit. British Journal of Psychiatry, 133, 137-142.

Faraone, S.V., Seidman, L. J., Kremen, W. S., et al (2000) Neuropsychologic functioning among the nonpsychotic relatives of schizophrenic patients: the effect of genetic loading. Biological Psychiatry, 48, 120 126.

DAGMAR VERSMISSEN, MA, INEZ MYIN-GERMEYS, MA, PhD, Department of Psychiatry and

Neuropsychology, South Limburg Mental Health Research and Teaching Network, EURON, Maastricht

University and Mondriaan Zorggroep, Section Social Cognition, Heerlen, the Netherlands; ILSE JANSSEN, MA, PhD Department of Psychiatry and Neuropsychology, South Limburg Mental Health Research and Teaching Network, EURON, Maastricht University, the Netherlands; NICOLAS FRANCK, MD, NICOLAS GEORGIEFF, MD, PhD, Centre Hospitalier Le Vinatier, Université Lyon I, Institut des Sciences Cognitives, Centre National de la Recherche Scientifique, France; JOOST a CAMPO, MD, PhD, Department of Psychiatry and

Neuropsychology, South Limburg Mental Health Research and Teaching Network, EURON, Maastricht University and Mondriaan Zorggroep, Section Social Cognition, Heerlen, the Netherlands; RON MENGELERS, Department of Psychiatry and Neuropsychology, South Limburg Mental Health Research and Teaching Network, EURON, Maastricht University, the Netherlands; JIM VAN OS, MD, PhD, Department of Psychiatry and Neuropsychology, South Limburg Mental Health Research and Teaching Network, EURON, Maastricht University, the Netherlands, and Division of Psychological Medicine, Institute of Psychiatry, London, UK, and Mondriaan Zorggroep, Section Social Cognition, Heerlen, the Netherlands; LYDIA KRABBENDAM, MA, PhD, Department of Psychiatry and Neuropsychology, South Limburg Mental Health Research and Teaching Network, EURON, Maastricht University, the Netherlands

Correspondence: Dr Lydia Krabbendam, Department of Psychiatry and Neuropsychology, Maastricht University, PO BOX 616 (VIJV), 6200 MD Maastricht, the Netherlands. Tel: ++31 43 3688682; fax: ++3I 43 3688689; email: I.krabbendam@sp.unimaas.nl

Fear, C., Sharp, H. \& Healy, D. (1996) Cognitive processes in delusional disorders. British Journal of Psychiatry, 168, 61-67.

Franck, N., Farrer, C., Georgieff, N., et al (200I) Defective recognition of one's own actions in patients with schizophrenia. American Journal of Psychiatry, I58, 454-459.

Franck, N., Posada, A., Pichon, S., et al (2005) Altered subjective time of events in schizophrenia. The Journal of Nervous and Mental Disease, 193, 350-353.

Frith, C. D., Blakemore, S. \& Wolpert, D. M. (2000) Explaining the symptoms of schizophrenia: abnormalities in the awareness of action. Brain Research. Brain Research Reviews, 31, 357-363.

Gould, R. A., Mueser, K. T., Bolton, E., et al (200I) Cognitive therapy for psychosis in schizophrenia: an effect size analysis. Schizophrenia Research, 48, 335-342.

Hanssen, M., Peeters, F., Krabbendam, L., et al (2003) How psychotic are individuals with nonpsychotic disorders? Social Psychiatry and Psychiatric Epidemiology, 38, 149-154.

Henquet, C., Krabbendam, L., Dautzenberg, J., et al (2005) Confusing thoughts and speech: source monitoring and psychosis. Psychiatry Research, 133, 5763.

Janssen, I., Krabbendam, L., Jolles, J., et al (2003) Alterations in theory of mind in patients with schizophrenia and non-psychotic relatives. Acto Psychiatrica Scandinavia, 108, II0-117.

Janssen, I., Versmissen, D., Campo, J. A., et al (2006) Attribution style and psychosis: evidence for an externalizing bias in patients but not in individuals at high risk. Psychological Medicine, 36, 771-778.

Johns, L. C., Rossell, S., Frith, C., et al (200I) Verba self-monitoring and auditory verbal hallucinations in patients with schizophrenia. Psychological Medicine, 31, 705-715

Johnson, M. K., Hashtroudi, S. \& Lindsay, D. S. (1993) Source monitoring. Psychological Bulletin, II4, 328.

Luteijn, F., Van der Ploeg, F. A. E. (1983) Handleiding Groninger Intelligentietest (GIT) (Manual Groningen Intelligence Test). Swets \& Zeitlinger.

Miller, P., Lawrie, S. M., Hodges, A., et al (2001) Genetic liability, illicit drug use, life stress and psychotic symptoms: preliminary findings from the Edinburgh study of people at high risk for schizophrenia. Social Psychiatry and Psychiatric Epidemiology, 36, 338-342.

Poulton, R., Caspi, A., Moffitt, T. E., et al (2000) Children's self-reported psychotic symptoms and adult schizophreniform disorder: a 15-year longitudinal study. Archives of General Psychiatry, 57, 1053-1058.

Schneider, K. (1959) Clinical Psychopathology (5th edn) (trans. M.W. Hamilton). Grune \& Stratton.

Spauwen, J., Krabbendam, L., Lieb, R., et al (2004) Does urbanicity shift the population expression of psychosis? Journal of Psychiatric Research, 38, 613-618.

Wing, J. K., Cooper, J. E. \& Sartorius, N. (1974) Measurement and Classification of Psychiatric Symptoms: An instruction manual for the PSE and Catego program. Cambridge University Press. 